\title{
DESolution of CD and CB macrocycles **
}

\author{
Jade A. McCune, Susanna Kunz, Magdalena Olesińska and Oren A. Scherman *
}

\begin{abstract}
Supramolecular chemistry utilizing the macrocyclic hosts cyclodextrins (CDs) and cucurbit[n]urils $(C B[n] s)$ is traditionally carried out in aqueous media, however, their solubility is typically poor, especially the family of cucurbit[n]urils. Through derivitization of these macrocycles their solubility can be augmented to enable enhanced solubility in water and in some organic solvents. The increase in solubility of these derivitized macrocycles allows for their use in a wider range of chemical environments and giving rise to myriad potential applications. We report the dissolution of parent $C D s(\alpha-, \beta$ - and $\gamma$-) and $C B[n] s$ ( $n$ $=6-8)$ in a in deep eutectic solvents (DES) showing dramatic enhanced solubility of the larger species in both families, $C B$ [7] and $C B$ [8] as well as $\beta$ - and $\gamma$ - CD, respectively. Furthermore, we show that the host-guest properties are maintained in this new solvation medium.
\end{abstract}

D eep eutectic solvents (DES) are a relatively new class of solvents first reported in 2002 by Abbot and coworkers. ${ }^{[1,2]}$ They share many similarities in their physicochemical properties with ionic liquids (ILs), however, have several notable differences. ${ }^{[3-5]}$ Made of biodegradable, cheap and non-toxic components, DES are an environmentally, economically and industrially more attractive alternative to ILs. In addition, their synthesis does not require an inert atmosphere and is considerably more straightforward. Typically, DES contain bulky ions that, on account of having a low lattice energy, form liquids at room temperature as a result of a decreased melting point. Delocalization of charge throughout the bulk liquid structure of the DES through the hydrogen bond network formed between the hydrogen bond donor and acceptor that causes this suppression in melting point. ${ }^{[4]}$

There are three main classes of DES, Type I are formed through $\mathrm{MCl}_{x}$ and a quaternary ammonium salt (where $\mathrm{M}$ is a metal), Type II through complexation of a hydrated metal halide and choline chloride $(\mathrm{ChCl})$ and Type III are formed

\footnotetext{
** This research was supported by the European Union (European Research Council Starting Grants ASPiRe 240629 (OAS), EPSRC departmental PhD studentship (EP/K503009/1 (JAM)) and Marie Curie FP7 SASSYPOL ITN (607602) programme (OAS \& MO).

* Jade A. McCune, Susanna Kunz, Magdalena Olesińska and Prof. O.A. Scherman

Melville Laboratory for Polymer Synthesis

Department of Chemistry, University of Cambridge

Lensfield Road, Cambridge, CB2 1EW, UK

E-mail: oas23@cam.ac.uk
}

Supporting information for this article is available on the WWW under or from the author. from $\mathrm{ChCl}$ and a hydrogen bond donor. ${ }^{[4]}$ As a result of the wide scope of compounds that fall within each type of DES, a broad range of solvents exists with tuneable physical and chemical properties. Type III DES, specifically those based upon ChCl-urea, Figure 1 (top), have shown the most promise in the solvation of a wide range of chemical entities including metal oxides, ${ }^{[1,2]}$ biopolymers ${ }^{[6,7]}$ and in the synthesis of materials for gas adsorption. ${ }^{[8,9]}$

Cyclodextrins (CDs) and cucurbit $[n]$ urils $(\mathrm{CB}[n] \mathrm{s})$ are two families of macrocyclic host molecules, Figure 1 (bottom). CDs are formed from cyclized glucopyranose units and are produced from the enzymatic degradation of starch whereas $\mathrm{CB}[n] \mathrm{s}$ are formed from the cyclization of $n$ glycoluril $(n=5-8,10)$ units joined by methylene bridges under acidic conditions. ${ }^{[10,11]}$ Despite having different chemical compositions they share many of the same properties, namely, they both act as host molecules capable of binding guest moieties inside their hydrophobic cavities. This binding event is driven, in part, by the release of conformationally restricted water molecules from the cavity. Both families are comprised of three main homologues, which increase in cavity diameter $\alpha-, \beta$ - and $\gamma-\mathrm{CD}$ and $\mathrm{CB}[6], \mathrm{CB}[7]$ and $\mathrm{CB}[8]$. The homologues of $\mathrm{CD}$ and $\mathrm{CB}[n]$ are approximately analogous in size and the nature of the guest molecules they can encapsulate. Within both families the cavity dimensions of the homologues vary, however, they all have a uniform height (CDs $7.8 \AA$ and $\mathrm{CB}[n] \mathrm{s} 9.1 \AA$ ). Similarly, the aqueous solubility of each homologue differs in both families, as detailed in Table 1.
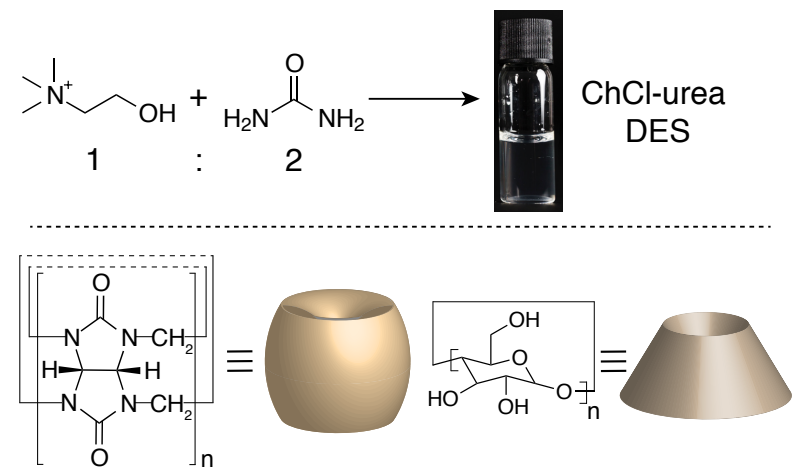

Figure 1 Top: Synthesis of ChCl-urea (1:2) DES. Bottom: Structure of $\mathrm{CB}[n] \mathrm{s}$ (left) and CDs (right).

CDs have, in general, much higher aqueous solubility compared to $\mathrm{CB}[n]$. $\mathrm{CB}[n]$ homologues where $n$ is odd, i.e. 
Table 1 Summary of aqueous solubility values of $\mathrm{CD}$ and $\mathrm{CB}[n]$ macrocycles.

\begin{tabular}{cc}
\hline Macrocycle & $\begin{array}{c}\text { Water solubility } \\
\mathbf{m g} / \mathbf{m L}\end{array}$ \\
\hline$\alpha-\mathrm{CD}$ & $129.5^{[12]}$ \\
$\beta-\mathrm{CD}$ & $18.4^{[12]}$ \\
$\gamma-\mathrm{CD}$ & $249.2^{[12]}$ \\
$\mathrm{CB}[6]$ & $<0.1$ \\
$\mathrm{CB}[7]$ & 4.4 \\
$\mathrm{CB}[8]$ & $<0.2$
\end{tabular}

$\mathrm{CB}[5]$ and $\mathrm{CB}[7]$ share similar solubilities as do the even homologues, i.e. $\mathrm{CB}[6]$ and $\mathrm{CB}[8]$. Both $\mathrm{CDs}$ and $\mathrm{CB}[n]$ s are generally insoluble in organic solvents and thus far have only been reported to be soluble in water. While CDs have been shown to be stable in alkaline media, they are readily hydrolyzed in acidic conditions, whereas $\mathrm{CB}[n] \mathrm{s}$ are reported to be stable in strong acidic environments.

To date, strategies to enhance the solubility of both macrocycles have been either through functionalization on their periphery, encapsulation of a guest molecule inside the cavity or through dissolution in an aqueous solution with another component such as $\mathrm{NaCl}$ for $\mathrm{CB}[n]$. The poor solubility of both $\mathrm{CDs}$ and $\mathrm{CB}[n] \mathrm{s}$ is a longstanding issue and enhancing the solubility in a solvent other than water whilst retaining host-guest properties has been a significant aim for many years. One rational for enhancing the solubility of a macrocycle is to increase the number of solvent-macrocycle interactions. Therefore, our strategy was based on increasing the number of solvent-macrocycle interactions through selection of a solvent, which not only had excellent solubilization properties but was also likely to interact with the macrocycle directly and thus achieve an enhancement of the overall solubility.

On account of many reports detailing their excellent solubilization properties, DES were considered as a potential alternative solvent. ${ }^{[3-5]}$ Moreover, the binding of $\mathrm{ChCl}$ to $\mathrm{CB}[7]$ has been reported in the literature by Macartney and co-workers. ${ }^{[13]}$ If the $\mathrm{CDs}$ and $\mathrm{CB}[n]$ were soluble in a DES in which in one component $(\mathrm{ChCl})$ is able to act as a weakly bound guest, we believed the number of solvent-macrocycle interactions would increase and $\mathrm{CDs}$ and $\mathrm{CB}[n] \mathrm{s}$ would exhibit enhanced solubility in DES compared to an aqueous environment.

First, we studied the solubility of $\alpha-\beta$ - and $\gamma$-CDs. In all cases we found that CDs were soluble in ChCl-urea DES and the solubilities of each CD were significantly enhanced, Table 2. For $\alpha$-CD the solubility was $>500 \mathrm{mg} \mathrm{CD} / \mathrm{mL}$ DES, however, in the cases of $\beta$ and $\gamma-\mathrm{CD}$, the solubility was found to be $>1 \mathrm{~g} / \mathrm{mL}$, which equates to $\mathrm{a} \approx 50 \mathrm{wt} \%$ solution of these CDs in the DES, a solubility that has never before been achieved. We postulate that this huge enhancement of solubility comes from the hydroxyl groups on the exterior of the $\mathrm{CD}$ being able to interact with the hydrogen bonded network of the DES, which results in the CDs being readily included within the bulk liquid structure at such high concentrations.

Table 2 Top: Table detailing the maximum solubility $(\mathrm{mg} / \mathrm{mL})$ of $\alpha-, \beta$ - and $\gamma-\mathrm{CD}$ and $\mathrm{CB}[n](n=6-8)$ in ChCl-urea DES and the enhancement factor relative to aqueous solution. Bottom: Vials showing ( $\mathrm{L}$ to R) $1 \mathrm{~g} / \mathrm{mL}$ of $\alpha-, \beta$ - and $\gamma-\mathrm{CD}$ and $\mathrm{CB}[6](1 \mathrm{mg} / \mathrm{mL})$, $\mathrm{CB}$ [7] $(17 \mathrm{mg} / \mathrm{mL})$ and $\mathrm{CB}[8](2 \mathrm{mg} / \mathrm{mL})$ dissolved in $1 \mathrm{~mL} \mathrm{ChCl}$ urea DES.

\begin{tabular}{ccc}
\hline Macrocycle & $\begin{array}{c}\text { DES solubility } \\
\text { mg/mL }\end{array}$ & $\begin{array}{c}\text { Enhancement } \\
\text { factor }\end{array}$ \\
\hline$\alpha-\mathrm{CD}$ & $>500$ & $>3.5$ \\
$\beta-\mathrm{CD}$ & $>1000$ & $>55$ \\
$\gamma-\mathrm{CD}$ & $>1000$ & $>4$ \\
$\mathrm{CB}[6]$ & 1.0 & 10 \\
$\mathrm{CB}[7]$ & 17 & 3.9 \\
$\mathrm{CB}[8]$ & 2.0 & 10 \\
\hline & & \\
\hline
\end{tabular}

Based on cavity size, price and (regulatory body) approval status $\beta$-CD is the most widely used $\mathrm{CD},{ }^{[14]}$ however, it has the poorest water solubility, Table 1 . The solubility enhancement in DES is greatest for $\beta$-CD at $>55 \mathrm{x}$, a substantial increase that will undoubtably have an impact on the industrial applications of $\beta$-CD.

Unlike CDs, values related to the solubility of $\mathrm{CB}[n] \mathrm{s}$ in different solvation environments is not as well documented in the literature. Therefore, the solubility of each $\mathrm{CB}[n](n=$ 6-8) homologue was initially studied in a variety of commonly used media, Table S1 (supporting information), as well as in ChCl-urea DES, Table 2. All CB $[n]$ homologues showed a significant solubility enhancement at room temperature in the $\mathrm{ChCl}$-urea DES than in water: $\mathrm{CB}$ [7] by almost a factor of $4(4.4 v s 15 \mathrm{mg} / \mathrm{mL})$ and $\mathrm{CB}[6]$ and $\mathrm{CB}[8]$ by a factor of $10(<0.1 v s 1.0 \mathrm{mg} / \mathrm{mL}$ and $<0.2 v s 2 \mathrm{mg} / \mathrm{mL}$, respectively) in ChCl-urea. It should be noted that the ChClurea DES is slightly basic ( $\mathrm{pH} 10$ at $20{ }^{\circ} \mathrm{C}^{[15]}$ ) and $\mathrm{CB}[n]$ have not been reported previously to exhibit any solubility in basic environments. Considering the enhanced solubility of $\mathrm{CB}[n]$ in acidic media, we also studied the solubility in an acidic DES, ChCl-TsOH and found that the solubility of $\mathrm{CB}[7]$ and $\mathrm{CB}[8]$ was even further enhanced (see supporting information Table S1).

The ability to dissolve both $\mathrm{CDs}$ and $\mathrm{CB}[n]$ homologues in a new medium with significant enhancement opens up a plethora of new and exciting opportunities. The poor solubility of both $\mathrm{CD}$ and $\mathrm{CB}[n]$ in water, and insolubility in most organic solvents, limits many applications either on account of low concentrations or poor solvent compatibility. From the solubilization of enhancements observed in Table 2 it was clear that the solvation environment of $\mathrm{ChCl}$-urea DES 
is unique. DES have a highly ordered liquid structure comprising many hydrogen bonding interactions, ${ }^{[1]}$ the enhanced solubility of $\mathrm{CB}[n]$ and especially $\mathrm{CDs}$ is likely due to the ability of this network to include the macrocycles within the liquid structure and stabilize such high amounts through further hydrogen bonding interactions. For the solvation and enhanced solubility of CDs and $\mathrm{CB}[n]$ in DES to be useful within the field, the host-guest properties of the macrocycles should ideally be preserved in this new solvation medium. However, probing the binding properties of macrocycles in DES is not as straightforward as in aqueous environments or traditional organic solvents.

On account of the highly ionic nature of the solvent, ${ }^{1} \mathrm{H}$ NMR cannot be utilized, similarly, the viscosity of $\mathrm{ChCl}$-urea is higher than water and thus isothermal titration calorimetry (ITC) cannot be readily conducted at room temperature. Cyclic voltammetry (CV) was attempted and indicated that some binding event occurred with both $\mathrm{CB}$ [7] and $\mathrm{CB}[8]$, Figures S8 and S9, respectively. Considering these limitations, we decided to probe binding further using UV/vis spectroscopy. To do so, we needed to identify a guest that had a characteristic UV/vis profile and would bind to both $\mathrm{CDs}$ and $\mathrm{CB}[n]$. The interaction of alkylviologens with both $\mathrm{CDs}$ and $\mathrm{CB}[n]$ is widely reported in the literature, therefore we chose methylviologen, the simplest and most studied alkylviologen.

In its dicationic form $\mathbf{M V}^{2+}$ is reported to bind 1:1 to both $\mathrm{CB}[7]^{[16,17]}$ and $\mathrm{CB}[8]^{[18,19]}$ but does not show any interaction with either $\beta$ - or $\gamma$-CD. ${ }^{[18]}$ However, methylviologen is a redox active molecule that can reversibly undergo two consecutive one electron reductions to form a monocationic radical species, $\mathbf{M V}{ }^{+}$or a fully reduced $\mathbf{M} \mathbf{V}^{0}$ species. In aqueous environments the monocationic radical species $\mathbf{M V}^{\cdot+}$ is reported to bind $1: 1$ with $\mathrm{CB}[7]^{[16,17]}$ and $\beta-\mathrm{CD}^{[20]}$ and $2: 1$ with $\mathrm{CB}[8]^{[19]}$ and $\gamma$-CD. ${ }^{[21]}$ Therefore, we decided to study the behavior of $\mathbf{M V}^{+}$in the presence of both CDs $(\beta$ - and $\gamma)$ and $\mathrm{CB}[n](n=7 \& 8)$ using UV/vis spectroscopy.

Initially, we studied the $1: 1$ complexes of $\mathbf{M V}^{+}$with $\mathrm{CB}$ [7] and $\beta$-CD. For $\mathrm{CB}$ [7] we saw that there was a supression of the absorbance at $609 \mathrm{~nm}$ upon the addition of CB[7], Figure S6 (supporting information) which indicates that binding of $\mathbf{M V}^{\cdot+}$ is occurring. ${ }^{[17]}$ Unfortunately, we were not able to observe this with $\beta-\mathrm{CD}$ as the required concentration was too high to allow us to obtain a UV/vis spectrum. Shown in Figure 2, the introduction of $\mathrm{CB}$ [8] to a $\mathbf{M V}^{\cdot+}$ solution resulted in a color change from blue to purple and a pronounced change in the UV/vis spectra. This color change is a result of the formation of the $\mathbf{M V}^{+}$dimer inside the CB[8] cavity. The $\mathbf{M V}^{+}$peak at $609 \mathrm{~nm}$ decreases in intensity and shifts to $605 \mathrm{~nm}$ upon addition of $\mathrm{CB}$ [8] with concomitant appearance of a peak at $557 \mathrm{~nm}$ corresponding to the formation of the dimer. Furthermore, the addition of a competitive guest for $\mathrm{CB}$ [8], 1-adamantylamine (Ada), to the solution results in a shift back to a blue solution as the $\mathbf{M V}^{+}$species have been displaced from the $\mathrm{CB}[8]$ cavity and the $\mathrm{CB}[8] \cdot\left(\mathbf{M V}^{+}\right)_{2}$ complex no longer exists. An anal- ogous experiment with $\gamma$-CD was also conducted, Figure S7, and $\gamma$-CD showed the same homoternary binding behavior as $\mathrm{CB}[8]$.

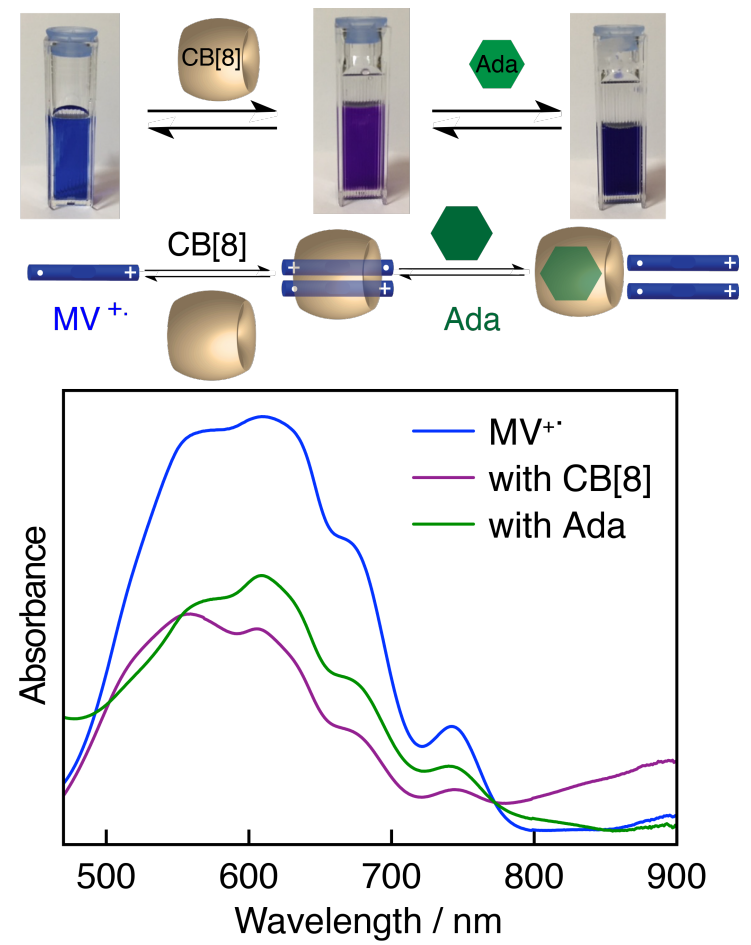

Figure 2 UV/vis spectra of $\mathbf{M V}^{\cdot+}$ in ChCl-urea DES (blue line), in the presence of $\mathrm{CB}[8]$ (purple line) and upon the addition of Ada (green line).

In conclusion, we have reported the dissolution of $\mathrm{CD}$ and $\mathrm{CB}[n]$ macrocycles in a novel class of deep eutectic solvents. The solubility of $\mathrm{CDs}(\alpha-, \beta$ - and $\gamma)$ and $\mathrm{CB}[n]$ $(\mathrm{n}=6-8)$ are found to be significantly enhanced in $\mathrm{ChCl}-$ urea compared to aqueous environments. The ability to dissolve both $\mathrm{CD}$ and $\mathrm{CB}[n]$ macrocycles in this new solvent at higher concentrations opens up many exciting possibilities as well as significantly enhancing the current industrial use of CDs (especially $\beta$-CD) and future use of $\mathrm{CB}[n] \mathrm{s}$. Finally, we demonstrate that $\mathrm{CD}$ and $\mathrm{CB}[n]$ retain their hostguest chemistry in the new solvation environment as shown through UV/vis spectroscopy with a model guest, $\mathbf{M V}^{\cdot+}$.

\section{References}

[1] A. P. Abbott, G. Capper, D. L. Davies, R. K. Rasheed, V. Tambyrajah, Chem. Commun. 2003, 70-71.

[2] A. P. Abbott, D. Boothby, G. Capper, D. L. Davies, R. K. Rasheed, J. Am. Chem. Soc. 2004, 126, 9142-9147.

[3] Q. Zhang, K. De Oliveira Vigier, S. Royer, F. Jerome, Chem. Soc. Rev. 2012, 41, 7108-7146.

[4] E. L. Smith, A. P. Abbott, K. S. Ryder, Chem. Rev. Nov. 2014, 114, 11060-11082.

[5] D. A. Alonso, A. Baeza, R. Chinchilla, G. Guillena, I. M. Pastor, D. J. Ramón, Eur. J. Org. Chem. 2016, 2016, 612-632. 
[6] A. P. Abbott, T. J. Bell, S. Handa, B. Stoddart, Green Chem. 2006, 8, 784-3.

[7] M. Sharma, C. Mukesh, D. Mondal, K. Prasad, RSC Adv. 2013, 3, 18149-7.

[8] S. Chen, J. Zhang, T. Wu, P. Feng, X. Bu, J. Am. Chem. Soc. Nov. 2009, 131, 16027-16029.

[9] J. Zhang, T. Wu, S. Chen, P. Feng, X. Bu, Agnew. Chem. Int. Ed. 2009, 48, 3486-3490.

[10] W. A. Freeman, W. L. Mock, N. Y. Shih, J. Am. Chem. Soc. 1981, 103, 7367-7368.

[11] J. Kim, I.-S. Jung, S.-Y. Kim, E. Lee, J.-K. Kang, S. Sakamoto, K. Yamaguchi, K. Kim, J. Am. Chem. Soc. 2000, 122, 540-541.

[12] E. Sabadini, T. Cosgrove, F. d. C. Egídio, Carbohydr. Res. Feb. 2006, 341, 270-274.

[13] I. W. Wyman, D. H. Macartney, Org. Biomol. Chem. 2010, 8, $253-$ 260.
[14] J. Szejtli, Med Res Rev 1994, 14, 353-386.

[15] F. S. Mjalli, O. U. Ahmed, Asia Pac. J. Chem. Eng. Feb. 2016, 11, 549-557.

[16] H.-J. Kim, W. S. Jeon, Y. H. Ko, K. Kim, Proc. Natl. Acad. Sci. U.S.A. 2002, 99, 5007-5011.

[17] W. Ong, M. Gómez-Kaifer, A. E. Kaifer, Org. Lett. May 2002, 4, 1791-1794.

[18] H.-J. Kim, J. Heo, W. S. Jeon, E. Lee, J. Kim, S. Sakamoto, K. Yamaguchi, K. Kim, Agnew. Chem. Int. Ed. 2001, 40, 1526-1529.

[19] W. S. Jeon, H.-J. Kim, C. Lee, K. Kim, Chem. Commun. 2002, 18281829.

[20] A Yasuda, H Kondo, M Itabashi, J Seto, J. Electroanal. Chem. 1986, 210, 265-275.

[21] A Yasuda, H Mori, J Seto, J. Appl. Electrochem 1987, 17, 567-573. 


\section{Entry for the Table of Contents (Please choose one layout only)}

Layout 1:
Supramolecular DESolution

Jade A. McCune, Susanna Kunz, Magdalena Olesińska and Oren A. Scherman

DESolution of CD and CB macrocycles

\section{Layout 2:}

\section{Catch Phrase:}

Author(s), Corresponding Author(s)* . Page Page

\section{Title Text}

Significantly high solubilities of CDs $(\alpha, \beta \& \gamma)$ and $\mathrm{CB}[n](\mathrm{n}=6,7, \&$ $8)$ are exhibited in a choline chloride $(\mathrm{ChCl})$-urea DES. $\beta$ \& $\gamma \mathrm{CDs}$ were soluble at $>1 \mathrm{~g} / \mathrm{mL}$ DES resulting in $50 \mathrm{wt} \%$ solutions, additionally, the solubility of $\mathrm{CB}[n]$ were also found to be significantly enhanced extending the range of $\mathrm{CB}$ solubility into alkaline media for the first time. The hostguest chemistry of these macrocycles is maintained upon dissolution in these highly ordered solvents.
$\begin{array}{llllll}a-C D & \beta-C D & \gamma-C D & C B[6] & C B[7] \quad C B[8]\end{array}$
Text for Table of Contents, max. 450 characters. 